\title{
Size-dependent surface energy density of typically fcc metallic nanomaterials
}

\author{
Cun Zhang, Yin Yao, Shaohua Chen* \\ LNM, Institute of Mechanics, Chinese Academy of Sciences, Beijing 100190, China
}

\section{A R T I C L E I N F O}

\section{Article history:}

Received 26 August 2013

Accepted 9 October 2013

Available online 5 November 2013

\section{Keywords:}

Nanomaterials

Surface energy density

Surface relaxation parameter

Lagrangian configuration

Eulerian configuration

\begin{abstract}
A B S T R A C T
The surface energy density of nano-sized structural elements exhibits an obvious size-dependent feature. To study this interesting phenomenon, atomistic calculations are carried out in the present paper for different face-centered-cubic (fcc) metallic nano-slabs. Lagrangian and Eulerian descriptions are adopted, respectively, in order to find the varying trends of surface energy densities in an initially un-deformed configuration and a current one. It is found that the Lagrangian surface energy density increases monotonically with an increase of the nano-slab's thickness in the former no matter what the surface orientation is; while the variation of the Eulerian one is indefinite. The surface relaxation parameters are further simulated for differently oriented surfaces, which gives a very good explanation for the differences between the Lagrangian and Eulerian surface energy densities. The results in this paper should be a useful supplement to theoretical studies on the surface/interface effect of nanomaterials.
\end{abstract}

(c) 2013 Elsevier B.V. All rights reserved.

\section{Introduction}

The mechanical, physical and chemical properties of a material will change significantly as its characteristic dimension is reduced to the nanometer range $[1,2]$. The main cause for this distinct sizedependent behavior is the effect of free surfaces [3,4]. Surfaces of solids possess atoms with fewer neighbors and consequently excess energies over atoms in the bulk, of which properties can greatly affect the overall performances of nanostructures having large surface-to-volume ratios, e.g., the elastic modulus [5], melting temperature [6] and thermal conductivity [7], etc.

Surface free energy is the most importantly physical attribute characterizing the nature of surface effects, which can be interpreted as a reversible work necessary to create a unit of new surface area [8]. The theoretical formulation of surface free energy within the framework of continuum mechanics has drawn many interests. As pointed out by Shuttleworth [9] and Vermaak et al. [10], surface energy is related to the deformation of solids' crystal faces, whose partial derivative with respect to the surface strain gives rise to a surface stress. Inspired by this basic idea, Gurtin and Murdoch [11,12] used a linearized constitutive law to formulate the surface free energy, based on which the surface elasticity theory was rigorously developed (G-M theory). Subsequently, Steigmann and Ogden [13] and Chhapadia et al. [14] modified the $\mathrm{G}-\mathrm{M}$ theory by introducing a curvature-dependent term into the constitutive law in order to analyze the bending and wrinkling

\footnotetext{
* Corresponding author. Tel.: +86 10 82543960; fax: +86 1082543977

E-mail address: chenshaohua72@hotmail.com (S. Chen).
}

effects of nanowires. Nix and Gao [15] derived the analytical expressions of surface energy density with Lagrangian and Eulerian descriptions. Huang and Wang [16] analyzed the corresponding terms under a finite deformation condition.

Since nanomaterials possess very small characteristic scales, the surface energy is very difficult to be measured experimentally. Atomistic computations, including ab initio and molecular dynamics (MD) approaches, are widely used in order to determine the surface energy of nanomaterials numerically. A database of surface energy densities for various face-centered-cubic (fcc) and bodycentered-cubic (bcc) metals with differently oriented faces were established by Vitos [17] and Shenoy [18], based on the first-principle and MD calculations, respectively. Similar works were done by Miller and Shenoy [19], Mi et al. [20] and Sheng et al. [21]. However, the size dependence of the surface energy density was not considered in these simulations. For nanomaterials with a large surface-to-volume ratio, the surface free energy density always shows a strong dependence on their characteristic scales $[9,10,22]$. A series of thermodynamic models were established to describe the size effect of surface energy density. Ouyang et al. [23,24] and Liang et al. [25] developed theoretical models by dividing the surface free energy density of nanomaterials into a structural part related to the surface relaxation and a chemical part originating from the surface dangling bonds. They predicted that the total surface energy density exhibits the same size-dependent behavior as its chemical part, both of which decrease with a decreasing characteristic length of a nanomaterial. All the works provide a clear and general insight into the basic physical and chemical natures of the surface energy density at nanoscale. 
However, studies on size dependencies of surface energy density are almost confined to the theoretical analysis and the relaxation parameters were not considered. What is the physical image of the Lagrangian and Eulerian descriptions of the surface energy density? Are there any differences of the size-dependent behavior between the two different descriptions?

In this paper, MD simulations are performed and the Lagrangian and Eulerian surface energy densities of several typically fcc metallic nano-slabs are calculated systematically for differently oriented surfaces as well as the surface relaxation parameters. All the MD results are explained by a simple theoretical analysis. The results in the present paper should be helpful for better understanding the size-dependent mechanisms of surface energy density at nanoscale.

\section{MD simulation model and definition of the surface energy density}

\subsection{An atomistic model}

The atomistic model of an fcc metallic nano-slab is shown in Fig. 1, which is cut from a bulk crystal. Periodic boundary conditions are applied in $x_{1}$ and $x_{2}$ directions. The lengths $L_{1}$ and $L_{2}$ in $x_{1}$ and $x_{2}$ directions are taken as about 20 cubic unit cells, of which values can hardly affect the surface energy densities of surfaces normal to $x_{1}$ and $x_{2}$ directions. The surface normal to $x_{3}$ direction is traction-free with a nano-scale thickness in $x_{3}$ direction. Surface effect in such a nano-slab should be induced by the surface normal to $x_{3}$ direction, whose surface energy density is to be calculated. To study the size dependence of surface energy density, the slab thickness $h$ varies from 2 to 20 unit cells (8-100 Á, roughly).

The atomic interactions abide by the embedded-atom-method (EAM) potential [26]. The relevant parameters involved in EAM potential are taken from Sheng et al. [21] where the parameters of each element were obtained by fitting the potential-energy surface (PES) to the high-precise first-principle calculations. In the embedded atom formalism, the total energy is written as [21]:

$E_{\text {tot }}=\sum_{i, j} \phi\left(r_{i j}\right)+\sum_{i} F\left(n_{i}\right)$ and $n_{i}=\sum_{j} \rho\left(r_{i j}\right)$

where $i$ and $j$ are indices that run over all atoms in the model, $r_{i j}$ is the distance between atoms $i$ and $j, F\left(n_{i}\right)$ is the embedding energy required to place atom $i$ in a local electron density $n_{i}$ and $\phi\left(r_{i j}\right)$ is a two-body potential between atoms $i$ and $j$. The summation is over the total number of atoms in the system. It should be noted that the total energy $E_{\text {tot }}$ in Eq. (1) is regarded as an additional energy with respect to the un-deformed lattice assumed zero energy per atom [18].

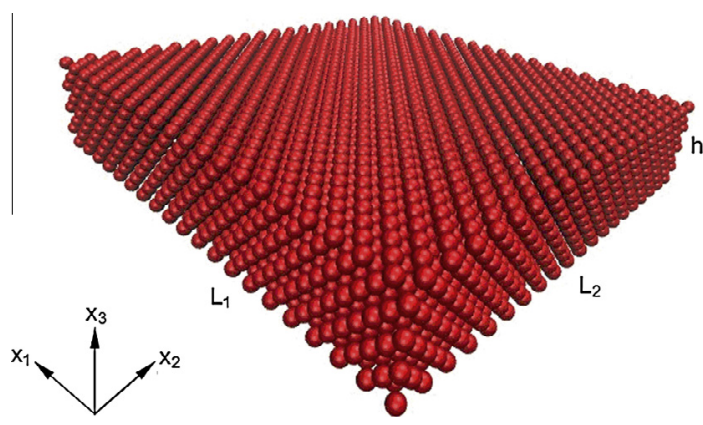

Fig. 1. Schematic of a nano-slab consisting of atoms for MD simulation, where $h$ is the thickness of the nano-slab in $x_{3}$ direction and periodic boundary conditions are used in $x_{1}$ and $x_{2}$ directions.
All MD simulations are performed using LAMMPS [27] with a time step of $1 \mathrm{fs}$. The system cut from a bulk crystal is relaxed at a constant atmospheric pressure ( 1 bar) and constant temperature $(1 \mathrm{~K})$ with Nose-Hoover thermostat and barostat. Note that NoseHoover barostat is only used in the periodic directions and the low temperature $1 \mathrm{~K}$ is chosen in order to avoid the effect of thermal disturbance on surface energies. When the equilibrium state of the simulation system is achieved at about $150 \mathrm{ps}$, the data will be recorded every $0.1 \mathrm{ps}$ in the next $50 \mathrm{ps}$.

\subsection{Surface energy densities in the reference and current configurations}

Consider a surface element $A_{0}$ taken from the initially un-deformed configuration $V_{0}$ as shown in Fig. 2. The element has planar array of atoms with $a_{01}, a_{02}$ and $N_{0}$ being the lattice spacing in the two principal directions of the surface and the total number of atoms in the surface, respectively. A Lagrangian coordinate system $\{123\}$ is imbedded in the surface and attached to the atoms [15]. As $V_{0}$ deforms into the current configuration $V_{1}$ (relaxation in our simulations), the initial element area $A_{0}$ becomes $A_{1}$ and the atomic distances in the two principal directions change from $a_{01}, a_{02}$ to $a_{1}$, $a_{2}$. According to Nix and Gao [15], the Lagrangian surface energy density $\phi_{L}$ in $V_{0}$ and the Eulerian one $\phi_{E}$ in $V_{1}$ can be expressed as follows,

$\phi_{L}=\frac{N_{0} g_{x S}^{1}}{A_{0}}, \quad \phi_{E}=\frac{N_{0} g_{x S}^{1}}{A_{1}}, \quad g_{x S}^{1}=g_{x S}^{0}+\frac{1}{2} \sum_{i=1}^{2} k_{i}\left(a_{i}-a_{0 i}\right)^{2}$

where $g_{x s}^{0}$ and $g_{x s}^{1}$ represent the excess free energy per surface atom in the reference and current configurations, respectively. $k_{i}(i=1,2)$ are spring constants in the two principal directions. Moreover, areas of the surface element in the reference and current configurations satisfy

$A_{1}=A_{0}\left(1+\varepsilon_{1}^{L}\right)\left(1+\varepsilon_{2}^{L}\right), \quad \varepsilon_{i}^{L}=\frac{a_{i}-a_{0 i}}{a_{0 i}},(i=1,2)$

where $\varepsilon_{1}^{L}$ and $\varepsilon_{2}^{L}$ are the Lagrangian surface strains in 1 and 2 directions. Combining Eqs. (2) and (3) yields

$\phi_{L}=\phi_{E}\left(1+\varepsilon_{1}^{L}\right)\left(1+\varepsilon_{2}^{L}\right)=\phi_{E} J_{s}$

where $J_{s}=\left(1+\varepsilon_{1}^{L}\right)\left(1+\varepsilon_{2}^{L}\right)$ is an Jacobean determinant characterizing the deformation between the reference and current configurations $[15,16]$.

\subsection{The Lagrangian and Eulerian surface energy densities in $M D$ simulations}

Similar to the previous studies [18,28], an initial nano-slab is created by placing all the atoms in the same positions as those in a bulk fcc lattice as shown in Fig. 1, which has an un-deformed lattice and is taken as the reference configuration. The area of the free surface normal to $x_{3}$ axis is denoted as $A_{\text {slab }}^{0}$. Relaxation will be carried out at a constant temperature $1 \mathrm{~K}$ with free boundaries in $x_{3}$ direction in order to minimize the total energy of the nano-slab. The relaxed nano-slab is in equilibrium and free of any external loads, which is called the current configuration. Deformation from the reference configuration to the current one is completely induced by the free surface relaxation and the area of the relaxed surface normal to $x_{3}$ direction changes to be $A_{\text {slab }}$. It should be noted that $A_{\text {slab }}^{0}$ consists of the same number of atoms as $A_{\text {slab }}$ and only the distance between each two atoms changes. The total energy of the deformed nano-slab then becomes $E_{\text {tot }}$ comparing to the initial energy $n E_{b u l k}$, where $E_{b u l k}$ is the free energy per atom in a bulk and $n$ is the total atom number in the nano-slab. The surface energy at nano-scale is then expressed as $E_{t o t}-n E_{b u l k}$, which 

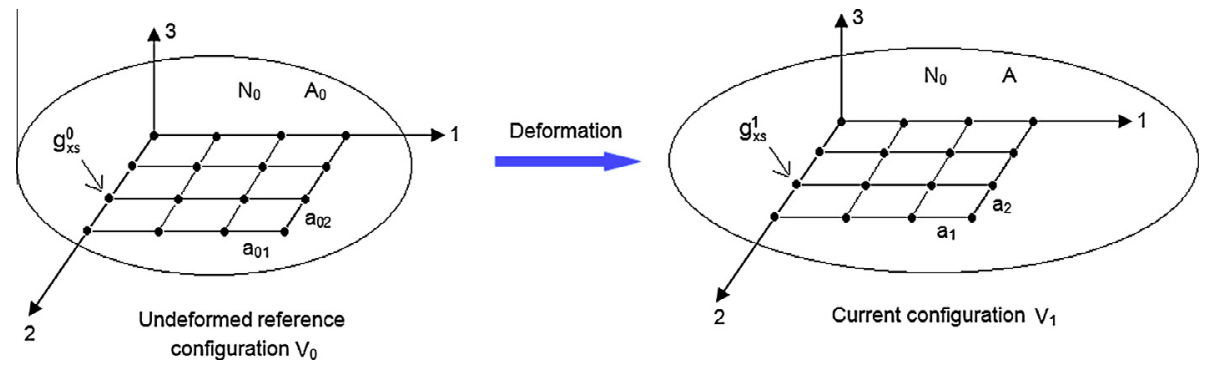

Fig. 2. Schematics of the crystal structure of a surface element transferring from the reference configuration to the current one due to relaxation deformation.

equals to $N_{0} g_{x s}^{1}$ in Eq. (2). In our MD simulations, the Lagrangian and Eulerian surface energy densities are defined as follows,

$\phi_{L}=\frac{E_{\text {tot }}-n E_{\text {bulk }}}{2 A_{\text {slab }}^{0}}, \quad \phi_{E}=\frac{E_{\text {tot }}-n E_{\text {bulk }}}{2 A_{\text {slab }}}$

In the previous simulation studies, only the Eulerian surface energy density $\phi_{E}$ was considered as a constant of nanomaterials $[18,29,30]$.

According to Eq. (3), area of the free surface in the reference and current configurations satisfies

$A_{\text {slab }}=A_{\text {slab }}^{0}\left(1+\varepsilon_{r 1}\right)\left(1+\varepsilon_{r 2}\right)=A_{\text {slab }}^{0} \lambda_{1} \lambda_{2}$

where the residual strains in 1 and 2 directions are $\varepsilon_{r 1}=\lambda_{1}-1$, $\varepsilon_{r 2}=\lambda_{2}-1$. The relations between the lattice constants before and after relaxation are $a_{r 1}=\lambda_{1} a_{01}, a_{r 2}=\lambda_{2} a_{02}$. $\lambda_{1}$ and $\lambda_{2}$ are surface relaxation parameters in the two principal directions. Substituting Eq. (6) into Eq. (5) leads to the relation between the Lagrangian surface energy density and the Eulerian one, which is consistent with Eq. (4).

\section{Results and discussion}

The Lagrangian and Eulerian surface energy densities of different fcc metallic nano-slabs with different thicknesses are calculated using Eq. (5). Three crystal orientations viz. (100), (110) and (111) of the upper and lower surfaces are studied. The fcc metals considered include $\mathrm{Ag}, \mathrm{Al}, \mathrm{Au}, \mathrm{Cu}$ and $\mathrm{Ni}$, whose EAM potentials have already been given by Sheng et al. [21].

The surface energy density of a bulk metal is first calculated when the thickness of the metallic slab is large enough. It is shown that both the Lagrangian and Eulerian surface energy densities are consistent well with those given by Sheng et al. [21]; furthermore, the Lagrangian and Eulerian surface energy densities are almost equal to each other for a sufficiently thick slab.

\subsection{Size effects of the Lagrangian surface energy density}

The Lagrangian surface energy densities for different fcc metallic nano-slabs with different thicknesses as well as different surface orientations are calculated. Fig. 3 presents the Lagrangian surface energy densities normalized by the corresponding bulk values $\phi_{L}^{B}$ as a function of the nano-slab's thickness for different metals. From Fig. 3, one can see that for all the calculated metals, the Lagrangian surface energy density increases with an increasing nano-slab's thickness no matter what surface orientation is. The Lagrangian surface energy density tends to be the bulk one as the thickness of the nano-slab is larger than $8 \mathrm{~nm}$ or so. Comparing Fig. $3(a-c)$, one can see that the Lagrangian surface energy densities of surfaces with different lattice orientations are different, too.

As we know that the Lagrangian surface energy density can be divided into a structural part $\phi_{L}^{\text {stru }}$ related to the surface relaxation and a chemical part $\phi_{L}^{\text {chem }}$ originating from the surface dangling bond energy [23]:

$\phi_{L}=\phi_{L}^{\text {chem }}+\phi_{L}^{\text {stru }}, \phi_{L}^{\text {stru }}=\frac{1}{2} \sum_{i=1}^{2} k_{i} \varepsilon_{r i}^{2}$

Assuming the crystal surface has a symmetrical structure with an identical distance between each two atoms in the two principal directions, which leads to the same relaxation parameters $\lambda_{1}=\lambda_{2}=\lambda$ in the two principal directions. Then, the surface residual strain $\varepsilon_{r 1}=\varepsilon_{r 2}=\left(a_{r}-a_{0}\right) / a_{0}=\lambda-1$. It is well known that the value of the relaxation parameter approaches to unity when the dimension of the structure becomes larger and larger, leading to a small absolute value of the surface residual strain, then the structural surface energy density $\phi_{L}^{\text {stru }}$ will decrease with an increasing size of the structure. However, it is exhibited that the present results of the total surface energy density increases with an increasing size of the structure. As a result, the chemical surface energy density should increase with an increasing size of the structure, which is well consistent with the conclusions made theoretically by Ouyang et al. [24] and Liang et al. [25]. The chemical surface energy density, as a dominant factor, governs the size-dependent behavior of the Lagrangian surface energy density of a nanostructure.

\subsection{Size effects of the Eulerian surface energy density}

The Eulerian surface energy densities for different fcc metallic nano-slabs with different thickness as well as different surface orientations are also calculated. Fig. 4(a-c) presents the Eulerian surface energy densities normalized by the corresponding bulk value $\phi_{E}^{B}$ as a function of the nano-slab's thickness for different metals. It is interesting to find that, for (100) and (110) crystal surfaces, the Eulerian surface energy densities of all the calculated fcc metals reduce with an increasing nano-slab's thickness, opposite to the varying trends of $\phi_{L}$. However, for the (111) oriented surface, the Eulerian surface energy densities of $\mathrm{Ag}, \mathrm{Al}, \mathrm{Cu}$ and $\mathrm{Ni}$ decrease, while the one of Au increases, with an increasing nano-slab's thickness. Therefore, in contrast to the monotonically size-dependent varying trend of the Lagrangian surface energy density, the varying trend of the Eulerian one is indefinite. The difference will be explained briefly in the following sub-section.

\subsection{Theoretical interpretations on the MD results}

The relation between the surface energy densities in a reference and current configurations is given in Eqs. (5) and (6), where the difference between the two terms is due to the surface relaxation parameters. It is interesting to find that the surface relaxation parameters depend also on the thickness of the nano-slab as shown in Fig. 5. Furthermore, the relaxation parameters are different for surfaces with different lattice orientations. Since both the 

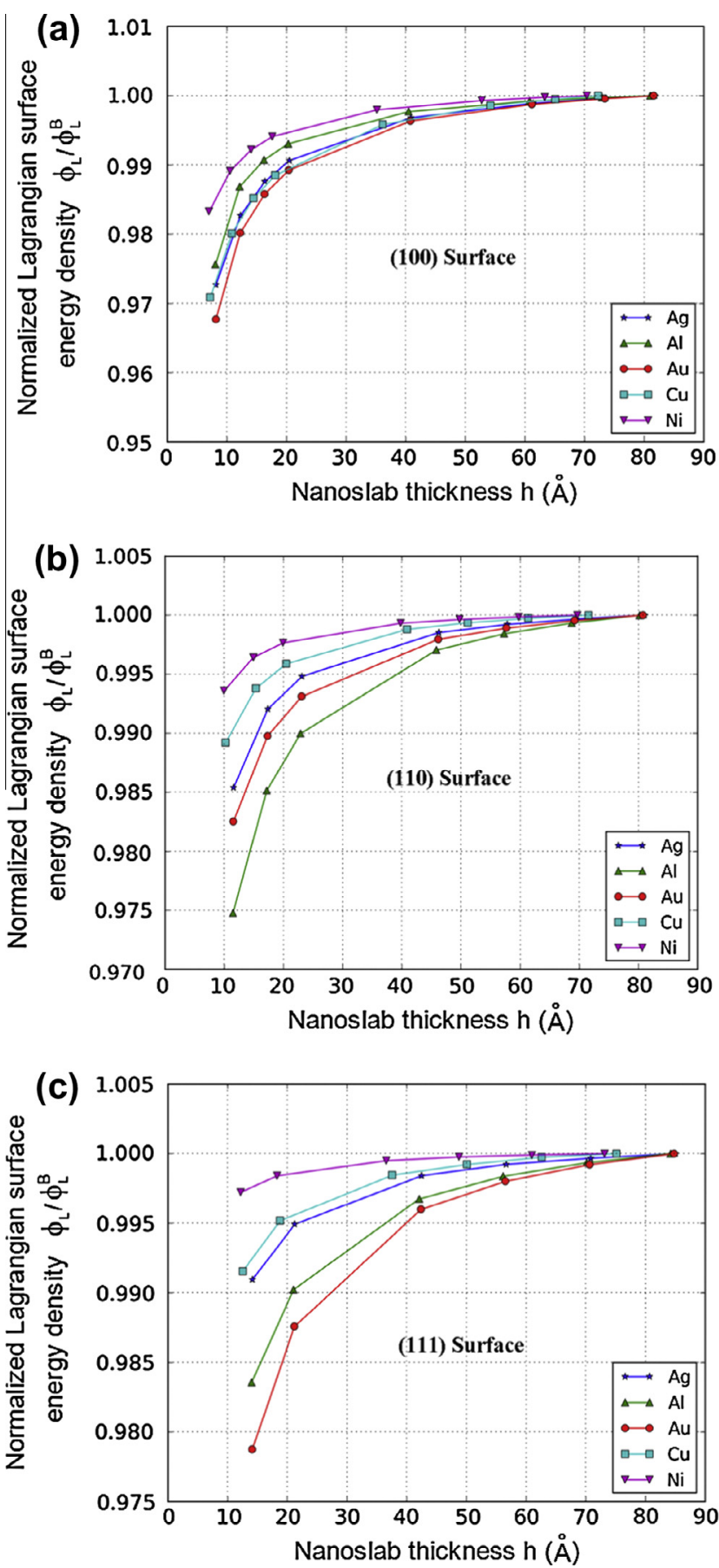

Fig. 3. The normalized Lagrangian surface energy densities of different metallic nano-slabs by the bulk one as a function of the nano-slab's thickness. (a) For the (100) crystal surface; (b) for the (110) crystal surface; (c) for the (111) crystal surface.

(100) and (111) surfaces possess a symmetrical lattice structure with an identical atomic distance between each two atoms as shown in the inset of Fig. 5(a) and (d), the surface relaxations in the two principal directions keep the same, i.e., $\lambda_{1}=\lambda_{2}=\lambda$. However, the surface relaxation parameter on the (110) surface exhibits an anisotropic feature due to the different atomic spacing in the principal bond directions as illustrated in Fig. 5(b) and (c). Both the surface relaxation parameters on (100) and (111) surfaces and that on (110) surface increase monotonically with an increasing thickness of the nano-slab. Then, the surface residual strain
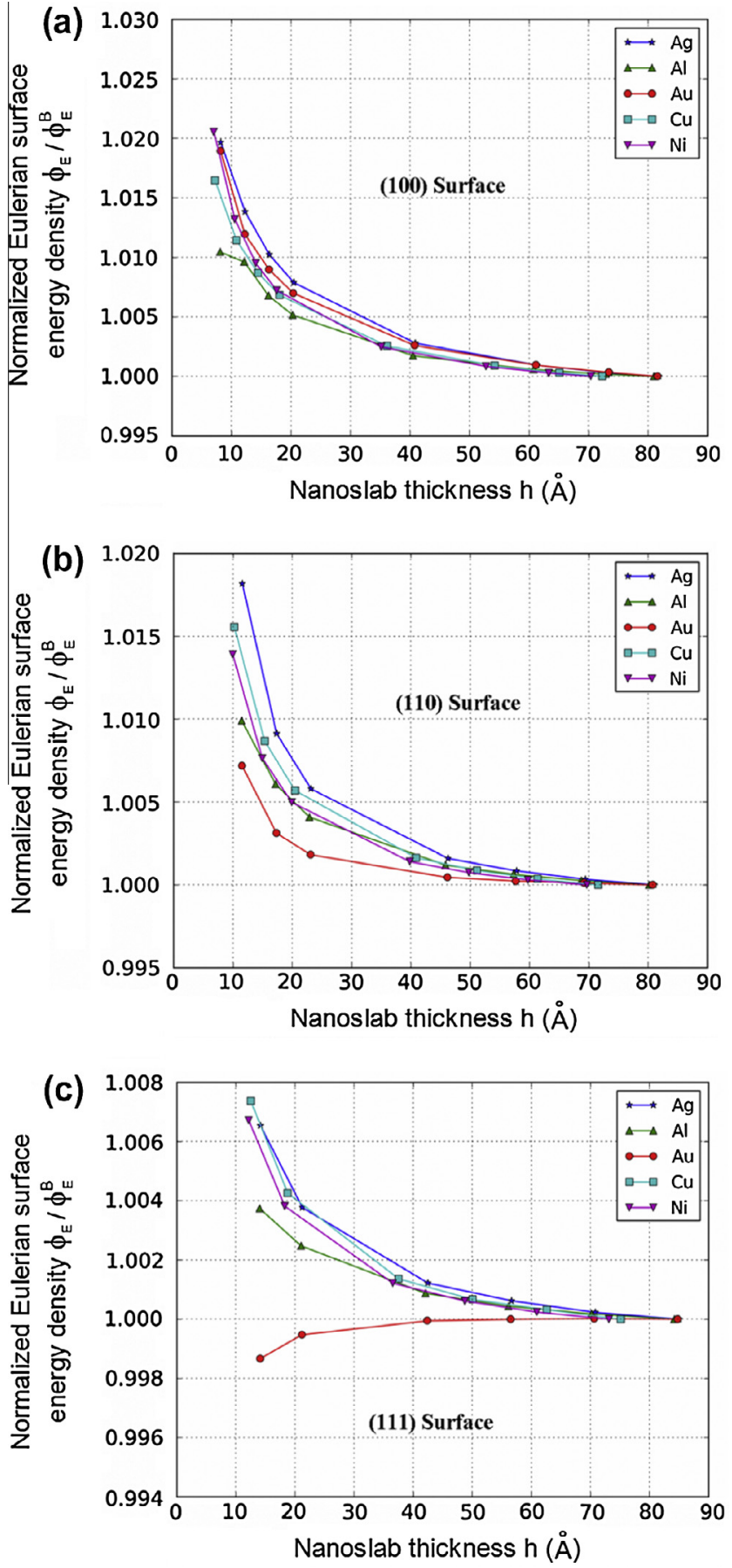

Fig. 4. The normalized Eulerian surface energy densities of different metallic nanoslabs by the bulk one as a function of the nano-slab's thickness. (a) For the (100) crystal surface; (b) for the (110) crystal surface; (c) for the (111) crystal surface.

$\varepsilon_{r i}=\lambda_{i}-1$ should be inversely proportional to the nano-slab's thickness, which agrees with the previous predictions for fcc metals $[23,28,31]$.

Combining Eqs. (4) and (6) leads to the following relation between the Lagrangian and Eulerian surface energy densities,

$\phi_{E}=\frac{\phi_{L}}{J_{S}}=\frac{\phi_{L}}{\left(1+\varepsilon_{r 1}\right)\left(1+\varepsilon_{r 2}\right)}=\frac{\phi_{L}}{\lambda_{1} \lambda_{2}}$

From Figs. 3 and 5, one can see that both the Lagrangian surface energy density $\phi_{L}$ and the product term of surface relaxation 

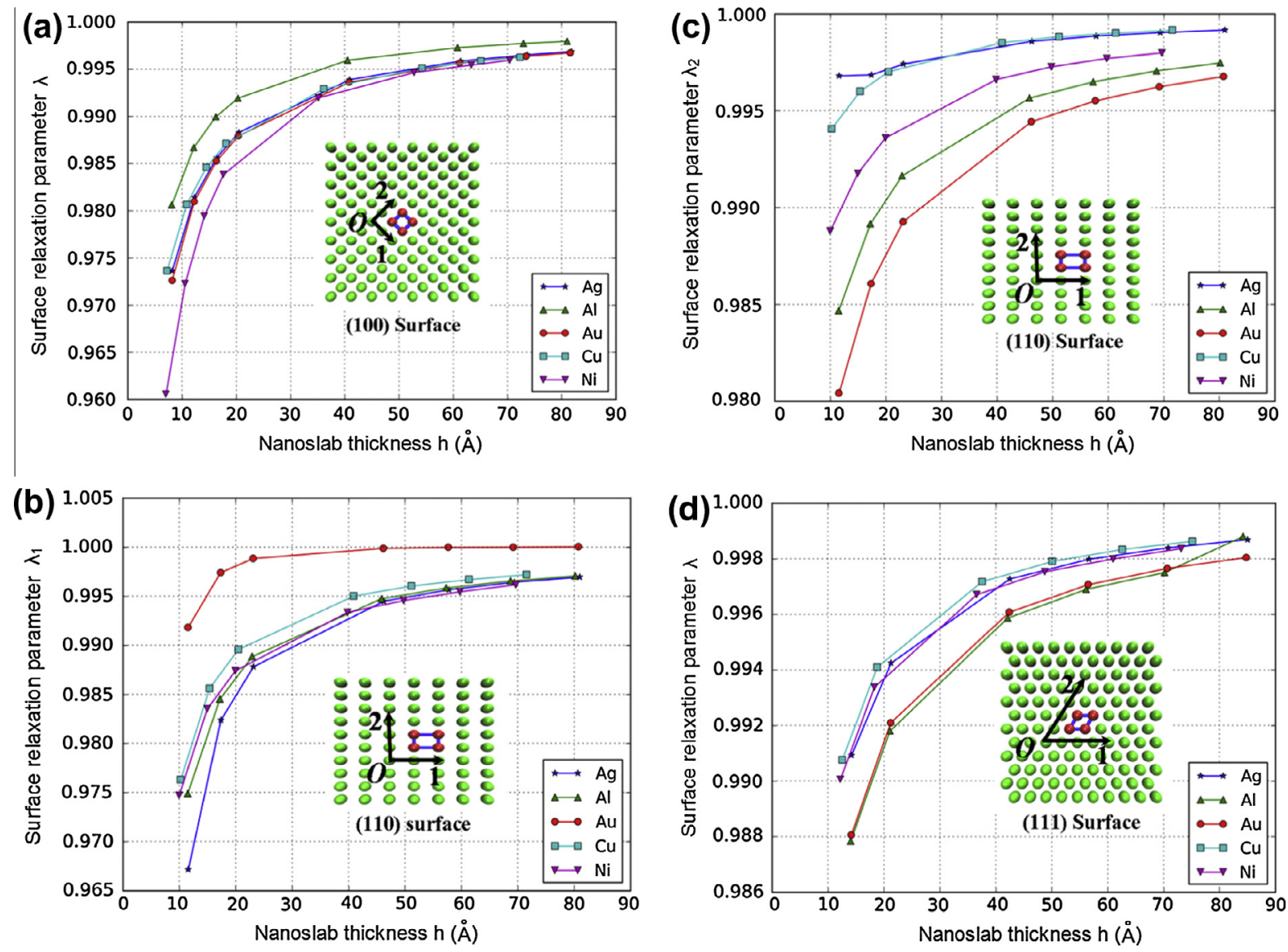

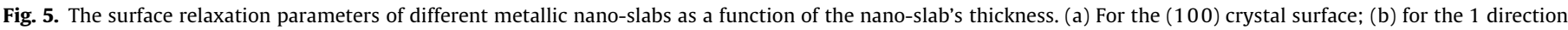
and (c) for the 2 direction of (110) crystal surface as shown in the inset; (d) for the (111) crystal surface.

parameters $\lambda_{1} \lambda_{2}$ increase with an increasing thickness of the nanoslab, which would induce an undetermined varying trend of the Eulerian surface energy $\phi_{E}$ with an increasing nano-slab's thickness. The analysis is consistent with the size-dependent behavior of $\phi_{E}$ as shown in Fig. 4.

\section{Conclusions}

Size dependence of the surface energy density of a nano-slab is investigated systematically in this paper with MD simulations and theoretical analysis. Both the Lagrangian and Eulerian descriptions are adopted to express the surface energy densities of surfaces with different lattice orientations, based on the initially un -deformed and currently deformed configurations, respectively. The results show that the Lagrangian surface energy density increases monotonically with an increasing nano-slab's thickness no matter what the surface orientation is; while the varying trend of the Eulerian one is indefinite and dependent of material element and the surface orientation. The surface relaxation parameters with different lattice orientations are further achieved. It is found that the surface relaxation parameters increase also with an increasing nano-slab's thickness. Considering the relations among the Lagrangian surface energy density, the Eulerian one and the surface relaxation parameters could give a reasonable explanation to the indefinite varying trend of the size-dependent feature of the Eulerian surface energy density. The present study incorporating the classical surface energy description within the framework of continuum mechanics into the atomistic simulation should be helpful not only for better understanding the physical mechanism of size-dependence of nanomaterials' surface energy density but also for establishing a novel theory considering the surface/interface effect for nano-structured materials.

\section{Acknowledgments}

The work reported here is supported by NSFC through Grants \#11125211, \#11021262 and the Nano-project (2012CB937500). The molecular simulations are carried out at Supercomputing Center of Chinese Academy of Sciences.

\section{References}

[1] H. Gleiter, Acta Mater. 48 (2000) 1-29.

[2] E. Roduner, Chem. Soc. Rev. 35 (2006) 583-592.

[3] H. Ibach, Surf. Sci. Rep. 29 (1997) 195-263.

[4] P. Muller, Surf. Sci. Rep. 54 (2004) 157-258.

[5] R. Dingreville, J. Qu, J. Mech. Phys. Solids 53 (2005) 1827-1854.

[6] L. Miao, V. Bhethanabotla, B. Joseph, Phys. Rev. B 72 (2005) 8717-8720.

[7] L.H. Liang, B. Li, Phys. Rev. B 73 (2006) 1-4.

[8] J.W. Gibbs, The Collected Works of J. Willard Gibbs, Longmans, Green, and Company, New York, 1928.

[9] R. Shuttleworth, Proc. R. Soc. Sec. A 63 (1950) 444-457.

[10] J.S. Vermaak, C.W. Mays, D. Kuhlmann-Wilsdorf, Surf. Sci. 12 (1968) 128-133.

[11] M.E. Gurtin, A.I. Murdoch, Arch. Rational Mech. Anal. 57 (1975) 291-323.

[12] M.E. Gurtin, A.I. Murdoch, Int. J. Solids Struct. 14 (1978) 431-440.

[13] D.J. Steigmann, R.W. Ogden, Proc. R. Soc. A 453 (1997) 853-877.

[14] P. Chhapadia, P. Mohammadi, P. Sharma, J. Mech. Phys. Solids 59 (2011) $2103-$ 2115.

[15] W.D. Nix, H. Gao, Scripta Mater. 39 (1998) 1653-1661.

[16] Z.P. Huang, J. Wang, Acta Mech. 182 (2006) 195-210.

[17] L. Vitos, Surf. Sci. 411 (1998) 186-202.

[18] V. Shenoy, Phys. Rev. B 71 (2005) 94104.

[19] R.E. Miller, V.B. Shenoy, Nanotechnology 11 (2000) 139-147.

[20] C. Mi, S. Jun, D. Kouris, S. Kim, Phys. Rev. B 77 (2008) 1-12. 
[21] H.W. Sheng, M.J. Kramer, A. Cadien, T. Fujita, M.W. Chen, Phys. Rev. B 83 (2011) 134118.

[22] Q. Jiang, D. Zhao, M. Zhao, Acta Mater. 49 (2001) 3143-3147.

[23] G. Ouyang, L.H. Liang, C.X. Wang, G.W. Yang, Appl. Phys. Lett. 88 (2006) 91914.

[24] G. Ouyang, X.L. Li, X. Tan, G.W. Yang, Nanotechnology 19 (2008) 045709.

[25] L.H. Liang, X.M. You, H.S. Ma, Y.G. Wei, J. Appl. Phys. 108 (2010) 84317.

[26] R.A. Johnson, Phys. Rev. B 37 (1988) 3924-3931.
[27] S. Plimpton, J. Comput. Phys. 117 (1995) 1-19.

[28] F.H. Streitz, R.C. Cammarata, K. Sieradzki, Phys. Rev. B 49 (1994) 10699.

[29] W. Luo, W. Hu, S. Xiao, J. Phys. Chem. C 112 (2008) 2359-2369.

[30] D.-P. Song, Y.-C. Liang, M.-J. Chen, Q.-S. Bai, Appl. Surf. Sci. 255 (2009) $5702-$ 5708.

[31] J. Diao, K. Gall, M.L. Dunn, J. Mech. Phys. Solids 52 (2004) 1935-1962. 